\title{
Synergistic platelet inhibition between Omega-3 and acetylsalicylic acid dose titration; an observational study
}

\author{
Harald Bagger ${ }^{1}$, Mattias Hansson ${ }^{1}$, Thomas Kander ${ }^{1,2}$ and Ulf Schött ${ }^{1,2^{*}}$ (D)
}

\begin{abstract}
Background: Omega-3 and acetylsalicylic acid (ASA) are two widely used "over-the-counter" drugs. Previous research has shown multiple electrode aggregometry (MEA) can detect ASA and varying Omega-3 platelet inhibiting effects. Synergistic platelet inhibiting effects of ASA and Omega-3 have been found using other methods than MEA. The aim of this study was to investigate the antiplatelet effects of Omega-3, and ASA synergism with MEA.
\end{abstract}

Methods: Ten healthy male volunteers ingested Omega-3 (1260 mg/day) for 5 days. MEA was used to analyse platelet function before and after Omega-3 intake. Aggregation was initiated using three different agonists and measured as area under the curve (AUC): adenosine diphosphate (ADP), thrombin receptor activating peptide (TRAP) and arachidonic acid (ASPI). Two concentrations of ASA were dose titrated ex vivo to 2 out of 3 ASPI test cells in order to measure synergism between Omega-3 and ASA.

Results: Following 5 days Omega-3 intake, ADP, TRAP and ASPI AUC did not change significantly. In vitro ASA before Omega-3 intake, reduced ASPI AUC < $30 \mathrm{U}$, indicating a strong platelet inhibiting effect. Below this AUC level, the 5 days Omega-3 intake increased ASPI-AUC with the ex vivo added low dose ASA $(P=0.02)$ and high dose ASA $(P=0.04)$.

Conclusions: No synergism between ASA and Omega-3 was found using the MEA ASPI test. The surprising increase in ASPI-AUC following Omega-3 intake and ex vivo ASA suggest that there are methodological issuses with the MEA ASPI test.

Trial registration: Trial registration ISRCTN78027929. Registered 19 May 2015.

Keywords: Coagulation, Acetylsalicylic acid, Omega-3

\section{Background}

Omega-3 fatty acids, primarily docosahexaenoic acid (DHA) and eicosapentaenoic acid (EPA), from fish oil are among the most frequently used dietary supplements, with $7.8 \%$ of U. S adults self-reporting frequent ingestion in 2012 [1]. Omega-3 has an established antiplatelet effect, and efforts have been made to examine through which specific mechanism(s) Omega-3 fatty

\footnotetext{
* Correspondence: ulf.schott@skane.se

'Institution of Clinical Science, Medical Faculty, Lund University, S-22185 Lund, Sweden

${ }^{2}$ Department of Anaesthesiology and Intensive Care, Skane University Hospital, S-22185 Lund, Sweden
}

acids affect platelet aggregation - with various results [2-5]. Dyerberg et al. [6] in 1979 suggested that high intake of Omega-3 fatty acids (fish oil) among Eskimos may cause increased bleeding. Definitive evidence is however nonexistent as to whether this antiplatelet effect on its own actually translates into increased clinical bleeding in patients $[2,7,8]$. A recent review identifies a platelet inhibiting effect of fish oil with no increased perioperative bleeding [9]. The platelet inhibiting effect of Omega-3 is probably weak, as the combination with clopidogrel does not to increase bleeding [10]. There are no reports on Omega-3 and stronger platelet inhibiting drugs. Omega- $3+$ warfarin does not increase bleeding 
[11]. There are no reports on Omega-3 and the new oral anticoagulants.

Aspirin (acetylsalicylic acid, ASA) is a widely used medication among adult patients for prevention of conditions such as cardiovascular disease, pain and inflammation. Between 2012 and 2015, more than 30\% of U.S. adults aged 40 years and older self-reported taking low dose ASA for prevention of cardiovascular disease [12]. Considering the fact that the patient group most likely to frequently ingest supplementary Omega-3, namely patients at risk for cardiovascular disease, are also indicated for ASA treatment, a significant number of patients likely use both substances. ASA should not usually be withdrawn prior to surgery as it is considered to be a weak platelet inhibiting drug [13], with the exception of neurosurgery [14]. The effect can be measured with MEA, but a platelet inhibiting effect does not always transfer into an increased perioperative bleeding [15].

A synergistic or additive antiplatelet interaction between Omega- 3 and ASA has been suggested by some studies $[4,16,17]$. A decrease in venous thromboembolism after total knee arthoplasty has been related to ASA+ Omega-3 synergism [18]. However, interactions between dietry supplements with antiplatelet effects and interactions with anticoagulation and antiplatelet drugs are not well studied [19].

The objective of the present study was to investigate the potential additional/synergistic effects of ASA + Omega-3 on platelet function with a point-of-care plateletet aggregometer, multiple electrode aggregometry (MEA), not studied before.

\section{Methods}

This observational non-randomized, non-blinded screening study was approved by the Regional Ethical Review Board, Lund (registration number 2010/482) and was conducted in accordance with the World Medical Association Code of Ethics (Helsinki Declaration 1975). The manuscript was prepared in accordance with the STROBE guidelines for observational studies. Consent was given both orally and in writing.

Ten healthy, male volunteers (aged 21-29, median 23) were recruited. No females were studied as female gender affects platelet function/platelet count and MEAresults [20]. Also Omega-3 has a stronger platelet inhibitory effect on males [21]. Exclusion criteria were recent intake of alcohol, anticoagulant or antithrombotic medicine. Subjects were instructed to ingest a standard recommended dose $(1260 \mathrm{mg})$ of two capsules of Omega-3 fish oil (Pharbio Omega-3 Forte ${ }^{\bullet}$, Pharbio Medical International AB, Solna, Sweden) per day, one in the morning and one in the evening, for 4 days. Day 5, 2 capsules were ingested $2 \mathrm{~h}$ before sampling (see below). A total of ten capsules $(12,600 \mathrm{mg})$ were ingested per volunteer during the study period. This daily dose represents an intake of $600 \mathrm{mg}$ eicosapentaenoic acid (EPA), $400 \mathrm{mg}$ docosahexaenoic acid (DHA), $60 \mathrm{mg}$ docosapentaenoic acid (DPA) and $200 \mathrm{mg}$ of other Omega-3. American Heart Association recommends at least $250 \mathrm{mg}$ /day of EPA + DHA [22] and FDA not more than $2 \mathrm{~g}$ per day from dietary supplements (www.fda.gov).

\section{Blood sampling}

Three ml of venous blood was drawn from an antecubital vein using a vacutainer system before and at the fifth day of Omega-3 treatment period. The volunteers rested 30 min before sampling and had had no stress or physical exertion during the morning, which can increase platelet aggregation [23]. Blood was collected in a $3.0 \mathrm{ml}$ Hirudin blood tube (Roche Diagnostics GMbH, Mannheim; Germany). The hirudin sprayed within the blood tube exerts its inhibitory effect on thrombin, without interfering with physiological calcium levels [24]. The blood samples were stabilized at room temperature for $30 \mathrm{~min}$, followed by MEA analysis within $3 \mathrm{~h}$ as recommended by the manufacturer [24] and Würtz et al. [25] to reduce test variability.

\section{Impedance aggregometry}

MEA analysis was performed using Multiplate Analyzer ${ }^{\circ}$ (Roche Diagnostics Scandinavia AB, Bromma, Sweden; V2.03.11). Through its 5 independent channels the Multiplate device is able to simultaneously measure the extent of platelet aggregation in each sample after the addition of different platelet agonists. Following the addition of platelet agonists, the change in electrical impedance between the two electrodes caused by the aggregation of platelets is detected. This change in electrical current is plotted in a graph over time and the results are expressed as area under the curve (AUC). The AUC represents the extent of platelet aggregation [26, 27].

Before analysis, $300 \mu \mathrm{l}$ of prewarmed $9 \mathrm{mg} / \mathrm{ml} \mathrm{NaCl}$ (B.Braun, Melsungen, Germany) was added to each test cell, followed by $300 \mu \mathrm{l}$ of whole blood. The whole blood and $\mathrm{NaCl}$ were incubated and stirred for $3 \mathrm{~min}$. Then $20 \mu \mathrm{l}$ of platelet agonists were added to their designated test cells. Three different platelet agonists were used, I) Adenosine diphosphate (ADP), $6.5 \mu \mathrm{M}$, an agonist which binds to ADP receptors and activates platelets through the release of endogenous ADP from dense granules, II) thrombin receptor activating peptide (TRAP), $(32 \mu \mathrm{M})$, a strong activator of platelets via the thrombin receptor, and III) ASPI (0.47$0.50 \mathrm{mM}$ ), which initiates platelet aggregation through the COX pathway [24]. COX is responsible for catalyzing the transformation of AA into thromboxane A2, a known potent activator of platelets [24]. 
Two concentrations of ASA were titrated to be added to two of three ASPI test cells, calculated to resemble real life blood concentrations with ASA treatment. 500 $\mathrm{mg}$ of ASA (Aspirin I.V. Bayer AG, Leverkusen, Germany) was diluted in $3 \mathrm{ml}$ of sterile water to ca. 170 $\mathrm{mg} / \mathrm{ml}$. One concentration, termed high dose ASA, was diluted 10 times to $17 \mathrm{mg} / \mathrm{ml}$. Another concentration, termed low dose ASA, was diluted 100 times to $1.7 \mathrm{mg} /$ $\mathrm{ml}$. Then $30 \mu \mathrm{l}$ of low respectively high dose ASA was added to ASPI test cells with a final concentration, of 0 , $8 \mathrm{mg} / \mathrm{ml}$ and $0.08 \mathrm{mg} / \mathrm{ml}$ respectively. The concentration of $0.08 \mathrm{mg} / \mathrm{ml}$ closely resembles the estimated physiological concentration of $0.1 \mathrm{mg} / \mathrm{ml}$ following intravenous treatment of $500 \mathrm{mg}$ ASA (the recommended treatment for acute myocardial infarction [28]. Intravenous (iv) aspirin ensures a better platelet inhibtion [29]. Our in vitro and ex vivo ASA protocol is more adherent to an iv approach.

Six minutes after addition of agonists the AUC of each test was recorded. ASPI-AUC was measured in three test cells: one without ASA and the other two in the test cells with added ASA. In total, the following 5 AUCs were analysed: ADP, TRAP, ASPI without ASA, ASPI with low and high dose ASA.

\section{Statistics}

Sample size was calculated using G*Power version 3.1, (Heinrich-Heine-Universität Düsseldorf, Germany) and was based on previous data on MEA-analyses before and after Omega-3 intake [26, 27]. With an alfa error probability of 0.05 and a power of 0.9 the sample size had to be 8 . We aimed at 10 volunteers to allow for sample failure.

The other statistical analyses were performed using GraphPad Prism version 8.3.0 (GraphPad Software Inc., La Jolla, CA). Gaussian distribution was tested using the D'Agostino \& Pearson test and the Shapiro-Wilk test. As Gaussian distribution was found, statistically significant changes in AUC following Omega-3 intake were analysed using a paired T test. Significance level was set at $P<0.05$. Potential outliers were analysed using the ROUT method.

\section{Results}

One volunteer was excluded from the study due to poor compliance. In total, nine volunteers were included in the statistical analyses. There were no significant changes in the MEA ADP, TRAP or ASPI-assays after Omega-3 intake (Fig. 1 and Table 1). In vitro ASA before Omega- 3 reduced ASPI AUC <30 U, indicating a strong platelet inhibiting effect. Below this AUC level, both ex vivo added low dose ASA $(P=0.02)$ and high dose ASA $(P=0.04)$ increased ASPI-AUC on the 5th day of Omega-3 intake (Fig. 2 and Table 1). A corresponding borderline nonsignificant increase in ASPI AUC $(P=0.07)$ within normal reference range could also be detected from before Omega-3 and on the 5th day (without ASA) (Table 1), indicating some non-inhibitory effect of Omega-3 on the ASPI-test. No outliers were found.

\section{Discussion}

The in vitro ASA effect on the MEA-ASPI prior to Omega-3 ingestion and the ex vivo ASA effect on the 5th day of Omega -3 intake indicated a strong platelet inhibition with an ASPI AUC $<30$ U. However, no additive or synergistic platelet inhibitory effect of 5 days peroral ingestion of Omega-3 was found using the MEA ASPI test.

The MEA ASPI test entails adding an AA agonist to the test cell blood sample, triggering platelet activation via the COX pathway. AA is a substrate of $\mathrm{COX}$, which transforms AA into thromboxane A2, a potent platelet activator. It is a commonly held view that Omega- 3 exerts its platelet function via inhibition of the AA-COX pathway. The basis for this view is that the Omega-3 fatty acids EPA and DHA competes with AA for COX, which might attenuate its action on AA. Also, following Omega-3 intake EPA and DHA are integrated into the phospholipid membrane, potentially at the expense of AA. This could also reduce AA-induced platelet aggregation [30]. If Omega-3 inhibits platelet aggregation via the AA-COX-axis, the ASPI AUC (representing AA induced platelet aggregation) would reasonably decrease following Omega-3 intake.

Our findings however contradict this view, as after Omega-3 intake the MEA ASPI AUC indicated an upward trend, although with no statistical significance, in the case of ASPI without ASA $(P=0.07)$. Significant increases in ASPI AUC after Omega-3 intake were seen with low dose ASA $(P=0.02)$ and with high dose ASA $(P=0.04)$ (Fig. 2). However, it must be stated that every individual value of ASPI AUC (without ASA) following Omega-3 intake was within normal MEA reference range [24, 26, 27], whereas some were below the reference value before Omega-3 intake (see below). Also, in the ASPI assays with both low and high dose ASA, all but 1 volunteer were below $30 \mathrm{U}$ AUC (the exception having an AUC of $32 \mathrm{U}$ in the ASPI+ASAx10 assay) following treatment. Less than $30 \mathrm{U}$ ASPI AUC has been defined as the cut-off value for strong inhibition of COX-1 by ASA [31]. The fact that all but one AUC were still below this cut-off of strong inhibition, heavily indicate that Omega-3 did not actually reverse this inhibition, and that the significant increases in AUC of ASPI+low and high dose ASA may be related to test variability, intra-individual variation in test response at repeted testing [32]. Whole blood Multiplate testing with hirudin have a higher variability than citrate and asprin 

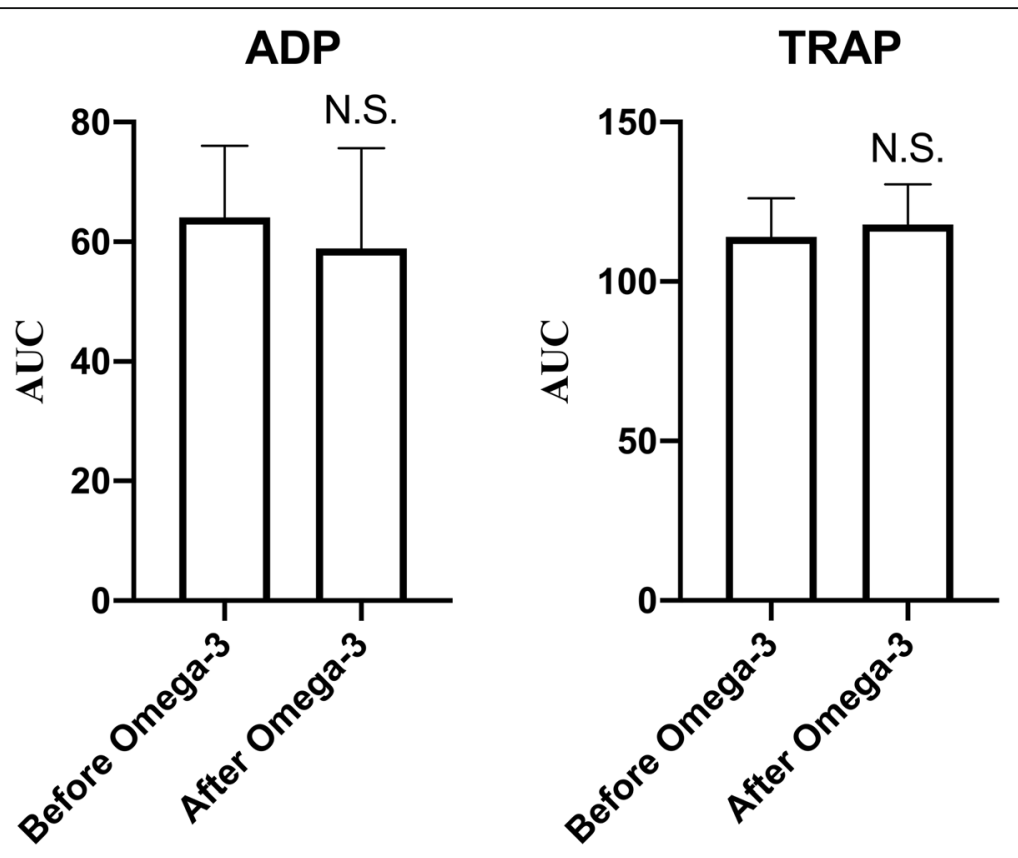

$\underline{\text { ADP }}$

$\underline{\text { TRAP }}$
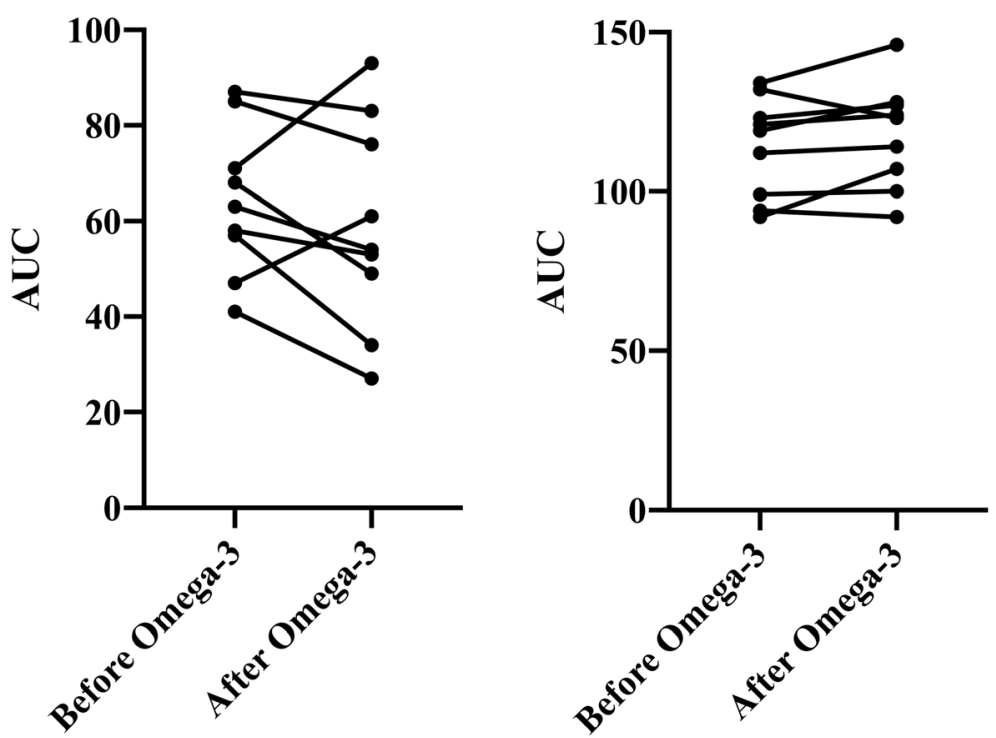

Fig. 1 Results from multiple electrode aggregometry (MEA) before and after Omega-3 intake, using thrombocyte receptor activating peptide (TRAP) and adenosine diphosphate (ADP). Results are presented as mean with 95\% confidence interval. N.S.: non-significant

Table 1 Results from multiple electrode aggregometry (MEA) assays

\begin{tabular}{lllll}
\hline MEA-ASSAY & Reference AUC range & Mean AUC before Omega-3 (95\% Cl) & Mean AUC after Omega-3 (95\% Cl) & $P-$ value \\
\hline ADP & $57-113$ & $64(52-76)$ & $59(42-76)$ & 0.32 \\
TRAP & $84-128$ & $114(102-126)$ & $118(105-130)$ & 0.15 \\
ASPI with no ASA & $71-115$ & $78(66-91)$ & $86(72-100)$ & 0.07 \\
ASPI with LOW DOSE ASA & N.A. & $12(9-15)$ & $18(13-22)$ & 0.02 \\
ASPI with HIGH DOSE ASA & N.A. & $14(9-19)$ & $21(17-26)$ & 0.04 \\
\hline
\end{tabular}

$\overline{A U C}$ area under the curve, $A D P$ adenosine diphosphate, TRAP thrombocyte receptor activating peptide, ASPII arachidonic acid 


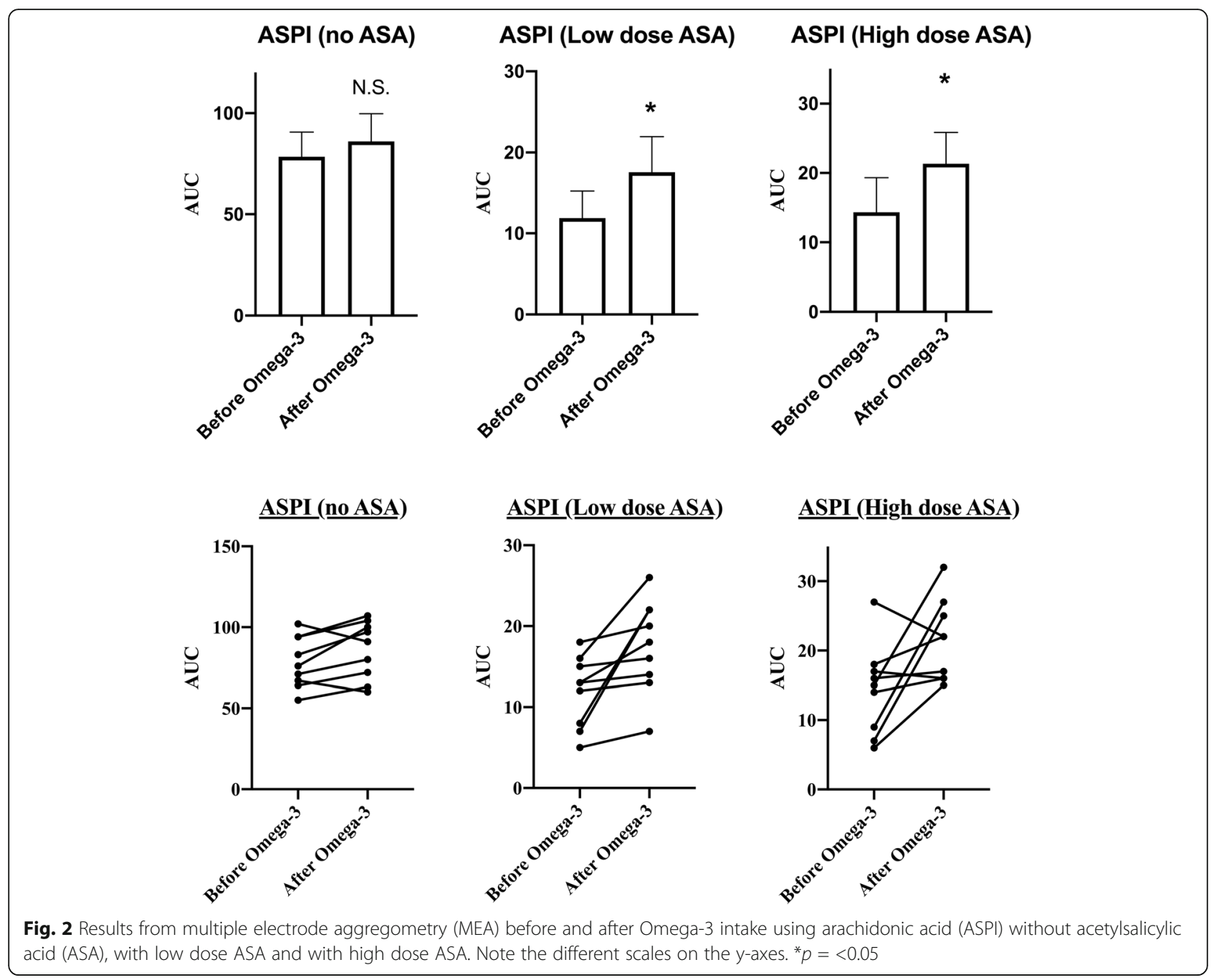

increase the test variability in hirudin anticoagulated ASPI-test [33]. With ongoing ASA treatment the MEA ASPI test has a coefficient of variation of $10 \%$ [34], but Pedersen et al. indentified a coefficient of variation (cv) of $8 \%$ increasing to $48 \%$ in the ASPI test before and after ASA treatment in healthy volunteers [35]. Such high ASPI test cvs have been corroborated by Peerschke et al. [33].

The lower ADP and ASPI AUC in some of the volunteers before Omega-3 intake may reflect unintentional over the counter drug intake or food/beverage intake with a similar effect, followed by a stricter regime during the study [32]. However only 2 of the volunteers increased in ADP response as compared to 7 in the ASPI response at the 5th day, hinting at a possible ASA/ NSAID effect, even though the volunteers negated this. Measuring thromboxane could have identified ASA/ NSAID effects [36].

Other studies examining the effects of Omega-3 intake using MEA have presented equivocal results. Kander et al. [26] found a significant decrease in ASPI AUC after adding Omega-3 in-vitro to blood from healthy volunteers. Mizia-Stec et al. [37] found a significant decrease of ASPI AUC in cardiovascular patients starting ASA and clopidogrel after percutaneous coronary inventions. A treatment group took Omega-3 - there were no differences in MEA (between Omega-3 patients and a control group without Omega-3 after 30 days in MEA (COL, TRAP, ASPI, ADP)), corroborating results of Watson et al. [10].

While our ASPI results do not necessarily support a procoagulant effect via the AA-COX pathway, they do indicate a lack of Omega-3 induced AA-COX antiplatelet effect. Recent studies also suggest this. Wada et al. [5] conducted a large experimental study comparing the effect of EPA versus AA on the COX pathway. EPA was found to be a very poor substrate for COX, suggesting that EPA has a limited ability to compete with AA as a COX substrate. Indeed, in a recent review article analysing multiple studies (including [5]) Wachira et al. [8] 
drew the conclusion that "there is little reason to believe that $\mathrm{n}-3$ fatty acids affect platelet biochemistry primarily via the effects on the COX pathway". Furthermore, while Gong et al. [4] found a synergistic effect on platelet aggregation between ASA and Omega-3 in mice, this was not affected by COX-1 knockdown. Aggregation was induced using ADP and collagen. Thus, while we in this study found no synergism or additive effect between ASA and Omega-3 in the AA-COX axis, such an effect may very well exist by other mechanisms. Also, Wachira et al. [8] suggests that metabolites of Omega-3 fatty acids produced through pathways other than the COXaxis could affect platelet function and thereby aggregation. Consequently, the highly dynamic and complex biochemistry of the multiple fatty acids present within platelets may affect our ability to isolate and determine the pro- or anti-platelet effect of individual fatty acid otherwise exerted in vivo [38]. Our results support the notion that Omega-3 works via other pathways than AA-inhibition.

In addition, several studies suggest that there could be other factors involved affecting the ASPI test and its AUC. In a recently published article, Ramström [39] investigated as to whether AA may cause lysis of blood cells, including platelets, and thereby affect ADPdependent platelet activation. Indeed, increasing concentrations of AA was found to cause cell lysis and release of ADP, suggesting that platelet activation may be influenced by the presence of ADP in the ASPI test. However, in the present study no efforts were made to examine this phenomenon, suggesting that our results may have been subject to its potential effect. Thus, raised awareness of potential effect by AA on the ASPI test is needed.

Moreover, Christiansen et al. [40] recently published an article set to investigate as to whether risk variants of the $\mathrm{AB} 0$ locus among patients with stable coronary artery disease (CAD) may affect platelet activation and aggregation. Indeed, an increased platelet aggregation assessed by MEA was found to be associated with the risk variant rs495828. Although not examined in the present study, these findings suggest that possible risk variants of the $\mathrm{AB} 0$ locus among the included volunteers could have had an impact on our results. However, as the study population of Christiansen et al. predominantly consisted of male patients with stable CAD and a mean age of 65 years, their findings may not be relevant to ours.

Denis et al. [41] found that mature platelets contain components of the spliceosome, although earlier considered to be limited to nucleated cells. Thus, these findings may indicate that platelets are able to splice pre-mRNA in response to exogenous signaling. Furthermore, Evangelista et al. [42] support this hypothesis of preserved functions and found evidence of signal and timedependent de novo synthesis of COX-1 in platelets, which may in part explain aspirin resistance. However, in the present study no sign of aspirin resistance among the included volunteers were observed. Also, a study conducted by Maree et al. [43] demonstrated that genetic variability in COX-1 may in part be responsible for the heterogeneous response observed in AA-induced platelets. In all, these studies indicate that platelets might contain preserved functions able to influence platelet aggregation, potentially explaining our surprising results.

The present study adds to the complexity of determining the effect of Omega-3 fatty acids on platelet aggregation. Of note, 5 out of 9 volunteers were below MEA ADP AUC reference range following Omega-3 intake, in line with the decreasing trend. In all, these findings are consistent with previous studies; in a metanalysis of 15 studies, Gao et al. [44] found a significant reduction in ADP-induced aggregation. As previously mentioned, the activation of platelets is complex and several components and pathways are thought to be involved. ADP is an important agonist which induces platelet aggregation through the membrane-bound $\mathrm{P}_{2} \mathrm{Y}_{12}$-receptor by indirectly increasing the cytosolic calcium-level $\left(\mathrm{Ca}^{2+}\right)$ and thereby enable the complex formation of GpIIb/IIIa [45]. However, some studies show the contrary, indicating a negligible effect on ADP-induced platelet aggregation in the presence of Omega-3 fatty acids $[27,46]$.

Perhaps more advanced laboratory technology should be used to study Omega- 3 effects on platelets. Omega-3 can reduce the von Willebrand factor (shear dependant platelet activator) [47]. We have previously studied Omega-3 effects with an automatic flow chamber technique $\left(\right.$ Cellix $\left.{ }^{\mathrm{Tm}}\right)$ with high shear stress, but failed to detect any platelet inhibiting effect [48]. Cohen et al. used electrophoretic quasi-elastic light scattering technology (EQELS) technology to study Omega-3 effects and found an increased negative resting platelet charge, e.g. decreased response to AA platelet activation [49]. They also used template bleeding time that increased with increasing doses from 1 to $8 \mathrm{~g}$ Omega-3. Interesting there were no changes in light transmission aggregometry, not even in patients on ASA/clopidogrel, corroborating our results that platelet aggregometry is not the adequate technique. The Omega-3 fraction EPA but not DHA can reduce platelet volume, an early sign of reduced platelet aggregation [50].

This study has several limitations. Only healthy young men were recruited as volunteers. As hypertriglyceridemia and coronary arterial disease have been shown to positively affect platelet responsiveness to Omega-3 supplementation [44], volunteers should have been more physiologically representative of the relevant patient 
population. While every volunteer denied recent intake of ASA or NSAIDs, intake prior to enrolment in the study likely varied. Measurement of thromboxane levels in blood or urine could have identified this, but needs special laboratory resources [36]. Also, convincing evidence from several studies suggest that diet may have considerable influence on platelet function, and thereby risk of cardiovascular disease as well [51-54]. In the current study the volunteers were not instructed to report dietary habits. However, in a recent study conducted by Krekels et al. [55] no significant effect on platelet aggregation after smoking, coffee, high-fat meals or physical exercise was observed. Thus, future studies examining as to whether diet may affect platelet aggregation, when measured by methods such as MEA are needed. Furthermore, the great inter-individual variation of volunteers in response to Omega-3 treatment, as is evident in the individual response graphs in Figs. 1 and 2, makes clear interpretation of results difficult. For instance, consider the ADP individual response graph with two volunteers strongly deviating from the otherwise clear trend of reduction, thus possibly skewing results. A greater sample size would limit this susceptibility and would increase statistical power.

Another limitation of this study is the fact that the 5day Omega-3 treatment period might have been insufficient for Omega-3 to actually exert its platelet effects, although signifcant increases in plasma and red cells Omega-3 fatty acid concentrations can be meassured already at day 7, but increasing over time [56]. Intravenous administration of Omega- 3 is rapidly taken up by platelets $(4 \mathrm{~h})$ and by erythrocytes (ERC) (improves deformability and blood flow [47]) after $48 \mathrm{~h}$ [57]. In vitro incubation with Omega-3 reduces thromboxane B2, prostaglandins F2,E 2 and D2 production from platelets already after $5 \mathrm{~min}$ [58]. Cao et al. [59] found an ingestion period of 8-20 weeks necessary to achieve an Omega-3 index (measuring total Omega-3 fatty acid concentration in plasma phospholipids and ERC membranes) recommended by von Schacky et al. for acardioprotective effect [60]. Different Omega-3 purified fractions also inhibit platelet aggregation with different agonists after varying oral intake periods. Platelet aggregation with DHA (6g/day) was reduced already after 6 days (collagen/ADP), but with EPA only platelet aggregation to collagen could be detected so early; for ADP it took 4 weeks of EPA intake to detect a reducing effect [60].

Bagge et al. [26] found a significant change in MEA ADP AUC following 7 days of daily $1260 \mathrm{mg}$ Omega-3 ingestion in a prospective pilot study. However, in a follow-up study with 10 days of daily $2520 \mathrm{mg}$ ingestion, no significant results were found [48]. While Cao et al. did not use MEA (unlike Bagge et al.), the far more direct method of measuring Omega-3 levels used by Cao et al. make their study more relevant in terms of discussing adequate Omega-3 treatment period for future studies. All in all, it is possible that significant results found after a treatment period shorter than that recommended by Cao et al. are merely chance findings that should be treated with caution. Recent research use higher doses of Omega-3 for longer periods and very advanced laboratory methods to evaluate cellular and antiinflammatory effects on cardiovascular health [60]. Although Omega-3 effects on platelets can be seen better in healthy volunteers, patients with risk factors should be studied - with higher doses [61, 62].

\section{Conclusions}

No synergism between ASA and Omega-3 was found using the MEA ASPI test. The surprising increase in ASPI-AUC following Omega-3 intake and ex vivo ASA suggest that there are methodological issuses with the MEA ASPI test. Other platelet laboratory methods should be used to study Omega-3 effects on platelet function.

\section{Abbreviations}

ASA: Acetylsalicylic acid; MEA: Multiple electrode aggregometry; AUC: Area under the curve; ADP: Adenosine diphosphate (ADP), t; TRAP: Thrombin receptor activating peptide; ASPI: Arachidonic acid; DHA: Docosahexaenoic; EPA: Eicosapentaenoic acid; COX: Cyclooxygenase; AA: Arachidonic acid

\section{Acknowledgements}

Not applicable.

\section{Authors' contributions}

US designed the study and sampled blood. $\mathrm{HB}$ and $\mathrm{MH}$ carried out all blood compatibility tests. $\mathrm{HB}, \mathrm{MH}$ and TK performed all statistical data analysis. $\mathrm{HB}$, $\mathrm{MH}$ and TK prepared the figures and tables. $\mathrm{HB}$ and $\mathrm{MH}$ wrote the first version of the manuscript. All authors contributed to the interpretation of the data and revised the manuscript critically and gave final approval of the version to be published. All authors agreed to be accountable for all aspects of the work

\section{Funding}

The study was funded by research funds from Lund University, to cover laboratory costs for the Master Thesis (Harald Bagger, Mattias Hansson) payed to Ulf Schött. 10.1186/s12906-020-02990-9 BCAM-D-20-00255R1 Open access funding provided by Lund University.

Availability of data and materials

Data will be available on reasonable request.

\section{Ethics approval and consent to participate}

The study was approved by the Regional Ethical Review Board, Lund (registration number 2010/482). All volunteers gave signed informed consent.

Consent for publication

Not applicable.

Competing interests

All authors declare that they have no competing interests. 
Received: 14 February 2020 Accepted: 16 June 2020

Published online: 02 July 2020

\section{References}

1. Clarke TC, Black LI, Stussman BJ, Barnes PM, Nahin RL. Trends in the use of complementary health approaches among adults: United States, 2002-2012. Hyattsville: National Center for Health Statistics; 2015. National Health Statistics Reports; No.79. Available from: https://www.cdc.gov/nchs/data/ nhsr/nhsr079.pdf. [cited 2019 Dec 18]

2. Svaneborg N, Kristensen SD, Hansen LM, Bullow I, Husted SE, Schmidt EB. The acute and short-time effect of supplementation with the combination of $\mathrm{n}-3$ fatty acids and acetylsalicylic acid on platelet function and plasma lipids. Thromb Res. 2002;105(4):311-6.

3. Smith WL. Cyclooxygenases, peroxide tone and the allure of fish oil. Curr Opin Cell Biol. 2005;17(2):174-82.

4. Gong Y, Lin M, Piao L, Li X, Yang F, Zhang J, et al. Aspirin enhances protective effect of fish oil against thrombosis and injury-induced vascular remodelling. Br J Pharmacol. 2015;172(23):5647-60.

5. Wada M, DeLong CJ, Hong YH, Rieke CJ, Song I, Sidhu RS, et al. Enzymes and receptors of prostaglandin pathways with arachidonic acid-derived versus eicosapentaenoic acid-derived substrates and products. J Biol Chem. 2007;282(31):22254-66

6. Dyerberg J, Bang HO. Haemostatic function and platelet polyunsaturated fatty acids in Eskimos. Lancet. 1979;2(8140):433-5.

7. Akintoye E, Sethi P, Harris WS, Thompson PA, Marchioli R, Tavazzi L, et al. Fish oil and perioperative bleeding. Circ Cardiovasc Qual Outcomes. 2018; 11(11):e004584.

8. Wachira JK, Larson MK, Harris WS. N-3 fatty acids affect haemostasis but do not increase the risk of bleeding: clinical observations and mechanistic insights. Br J Nutr. 2014;111(9):1652-62.

9. Munk Begtrup K, Engel Krag A, Hvas AM. No impact of fish oil supplements on bleeding risk: A systematic review. Dan Med J. 2017;64(5):A5366.

10. Watson PD, Joy PS, Nkonde C, Hessen SE, Karalis DG. Comparison of bleeding complications with omega-3 fatty acids + aspirin + clopidogrel-versus--aspirin +clopidogrel in patients with cardiovascular disease. Am J Cardiol. 2009;104(8):1052-4

11. Pryce R, Bernaitis N, Davey AK, Badrick T, Anoopkumar-Dukie S. The Use of Fish Oil with Warfarin Does Not Significantly Affect either the International Normalised Ratio or Incidence of Adverse Events in Patients with Atrial Fibrillation and Deep Vein Thrombosis: A Retrospective Study. Nutrients. 2016:8(9):578 Published 2016 Sep 20.

12. Stuntz $M$, Bernstein $B$. Recent trends in the prevalence of low-dose aspirin use for primary and secondary prevention of cardiovascular disease in the United States, 2012-2015. Prev Med Rep. 2017:5:183-6.

13. Devereaux PJ, Mrkobrada M, Sessler DI, Leslie K, Alonso-Coello P, Kurz A, et al. POISE-2 investigators. Aspirin in patients undergoing noncardiac surgery. N Engl J Med. 2014;370(16):1494-503.

14. Václavík J, Táborský M. Antiplatelet therapy in the perioperative period. Eur J Intern Med. 2011;22(1):26-31.

15. Petricevic M, Konosic S, Biocina B, Dirkmann D, White A, Mihaljevic MZ, et al. Bleeding risk assessment in patients undergoing elective cardiac surgery using ROTEM $\left(^{\oplus}\right)$ platelet and multiplate $\left(^{(}\right)$impedance aggregometry. Anaesthesia. 2016;71(6):636-47.

16. Thorngren M, Gustafson A. Effects of 11-week increases in dietary eicosapentaenoic acid on bleeding time, lipids, and platelet aggregation Lancet. 1981:2(8257):1190-3.

17. Lev El, Solodky A, Harel N, Mager A, Brosh D, Assali A, et al. Treatment of aspirin-resistant patients with omega-3 fatty acids versus aspirin dose escalation. J Am Coll Cardiol. 2010;55(2):114-21.

18. Bonutti PM, Sodhi N, Patel YH, Sultan AA, Khlopas A, Chughtai M, et al. Novel venous thromboembolic disease (VTED) prophylaxis for total knee arthroplasty-aspirin and fish oil. Ann Transl Med. 2017;5(Suppl 3):S30

19. Olas B. Dietary supplements with antiplatelet activity: A solution for everyone? Adv Nutr. 2018;9(1):51-7

20. Berlin G, Hammar M, Tapper L, Tynngård N. Effects of age, gender and menstrual cycle on platelet function assessed by impedance aggregometry. Platelets. 2019;30(4):473-9.

21. Phang M, Sinclair AJ, Lincz LF, Garg ML. Gender-specific inhibition of platelet aggregation following omega-3 fatty acid supplementation. Nutr Metab Cardiovasc Dis. 2012;22(2):109-14.
22. Siscovick DS, Barringer TA, Fretts AM, et al. Omega-3 polyunsaturated fatty acid (fish oil) supplementation and the prevention of clinical cardiovascular disease: A science advisory from the American Heart Association. Circulation. 2017;135(15):e867-84.

23. Aldemir $\mathrm{H}$, Kilic $\mathrm{N}$. The effect of time of day and exercise on platelet functions and platelet-neutrophil aggregates in healthy male subjects. Mol Cell Biochem. 2005;280(1-2):119-24.

24. Diapharma. Multiplate Package Insert Compendium [pamphlet on the Internet]. West Chester: DiaPharma Group; 2015. Available from: https:// diapharma.com/wp-content/uploads/2016/07/Multiplate_Package_Inserts_ R5_ML-02-00011-.pdf. [cited 2020 Jan 05].

25. Würtz M, Hvas AM, Christensen KH, Rubak P, Kristensen SD, Grove EL. Rapid evaluation of platelet function using the multiplate ${ }^{\oplus}$ analyzer. Platelets. 2014;25(8):628-33

26. Bagge A, Schott $U$, Kander T. Effects of naturopathic medicines on multiplate and ROTEM: a prospective experimental pilot study in healthy volunteers. BMC Complement Altern Med. 2016;16:64.

27. Kander T, Lindblom E, Schott U. Dose-response effects of omega-3 on platelet aggregation: an observational study. J Int Med Res. 2018;46(12): 5074-82.

28. FASS Vårdpersonal. Trombyl. FASS; 2020. Available from:https://www.fass.se/ LIF/product?ignoreHandleMobile $=$ true\&nplld=19910906000043\&userType $=$ 0\&docType=3\&scrollPosition=645. [cited 2020-01-06].

29. Bach J, Kammerer I, Isgro F, Haubelt H, Vogt A, Saggau W, et al. The impact of intravenous aspirin administration on platelet aspirin resistance after onpump coronary artery bypass surgery. Platelets. 2009;20(3):150-7.

30. Adili R, Hawley M, Holinstat M. Regulation of platelet function and thrombosis by omega-3 and omega- 6 polyunsaturated fatty acids. Prostaglandins Other Lipid Mediat. 2018;139:10-8.

31. von Pape KW, Dzijan-Horn M, Bohner J, Spannagl M, Weisser H, Calatzis A. Control of aspirin effect in chronic cardiovascular patients using two whole blood platelet function assays. PFA-100 and multiplate. Hamostaseologie. 2007;27(3):155-60

32. Miller CH, Rice AS, Garrett K, Stein SF. Gender, race and diet affect platelet function tests in normal subjects, contributing to a high rate of abnormal results. Br J Haematol. 2014;165(6):842-53.

33. Peerschke El, Castellone DD, Stroobants AK, Francis J. Reference range determination for whole-blood platelet aggregation using the multiplate analyzer. Am J Clin Pathol. 2014;142(5):647-56.

34. Jámbor C, Weber CF, Gerhardt K, et al. Whole blood multiple electrode aggregometry is a reliable point-of-care test of aspirin-induced platelet dysfunction. Anesth Analg. 2009;109(1):25-31.

35. Pedersen SB, Grove EL, Nielsen HL, Mortensen J, Kristensen SD, Hvas AM. Evaluation of aspirin response by multiplate whole blood aggregometry and light transmission aggregometry. Platelets. 2009;20(6):415-20.

36. Vesterqvist $\mathrm{O}$, Schött U, Berséus O, Axelsson K, Gréen K. In vivo production of thromboxane and prostacyclin in patients following total hip arthroplasty. Scand J Clin Lab Invest. 1988;48(3):233-9.

37. Mizia-Stec K, Mizia M, Haberka M, Lasota B, Gieszczyk-Strozik K, Chmiel A, et al. N-3 polyunsaturated fatty acids do not influence the efficacy of dual antiplatelet therapy in stable angina pectoris patients after percutaneous coronary intervention. Cardiol J. 2013;20(5):478-85.

38. Morita I, Takahashi R, Saito Y, Murota S. Stimulation of eicosapentaenoic acid metabolism in washed human platelets by $12-$ hydroperoxyeicosatetraenoic acid. J Biol Chem. 1983;258(17):10197-9.

39. Ramstrom S. Arachidonic acid causes lysis of blood cells and ADPdependent platelet activation responses in platelet function tests. Platelets. 2019;30(8):1001-7.

40. Christiansen MK, Larsen SB, Nyegaard M, Neergaard-Petersen S, Wurtz M, Grove EL, et al. The ABO locus is associated with increased platelet aggregation in patients with stable coronary artery disease. Int J Cardiol. 2019;286:152-8

41. Denis MM, Tolley ND, Bunting M, Schwertz $H$, Jiang $H$, Lindemann $S$, et al. Escaping the nuclear confines: signal-dependent pre-mRNA splicing in anucleate platelets. Cell. 2005;122(3):379-91.

42. Evangelista V, Manarini S, Di Santo A, Capone ML, Ricciotti E, Di Francesco L, et al. De novo synthesis of cyclooxygenase-1 counteracts the suppression of platelet thromboxane biosynthesis by aspirin. Circ Res. 2006;98(5):593-5.

43. Maree AO, Curtin RJ, Chubb A, Dolan C, Cox D, O'Brien J, et al. Cyclooxygenase-1 haplotype modulates platelet response to aspirin. J Thromb Haemost. 2005;3(10):2340-5. 
44. Gao LG, Cao J, Mao QX, Lu XC, Zhou XL, Fan L. Influence of omega-3 polyunsaturated fatty acid-supplementation on platelet aggregation in humans: a meta-analysis of randomized controlled trials. Atherosclerosis. 2013;226(2):328-34.

45. Jin J, Kunapuli SP. Coactivation of two different G protein-coupled receptors is essential for ADP-induced platelet aggregation. Proc Natl Acad Sci U S A. 1998;95(14):8070-4.

46. Goodnight SH Jr, Harris WS, Connor WE. The effects of dietary omega 3 fatty acids on platelet composition and function in man: a prospective, controlled study. Blood. 1981;58(5):880-5.

47. DiNicolantonio JJ, OKeefe J. Importance of maintaining a low omega-6/ omega-3 ratio for reducing platelet aggregation, coagulation and thrombosis. Open Heart. 2019;6(1):e001011.

48. Bagge A, Schött U, Kander T. High-dose omega-3 fatty acids have no effect on platelet aggregation or coagulation measured with static and flowbased aggregation instruments and Sonoclot; an observational study in healthy volunteers. Scand J Clin Lab Invest. 2018;78(7-8):539-45.

49. Cohen MG, Rossi JS, Garbarino J, Bowling R, Motsinger-Reif AA, Schuler C, et al. Insights into the inhibition of platelet activation by omega-3 polyunsaturated fatty acids: beyond aspirin and clopidogrel. Thromb Res. 2011;128(4):335-40.

50. Park Y, Harris W. EPA, but not DHA, decreases mean platelet volume in normal subjects. Lipids. 2002;37(10):941-6.

51. McEwen BJ. The influence of diet and nutrients on platelet function. Semin Thromb Hemost. 2014;40(2):214-26.

52. Dauchet $L$, Amouyel P, Hercberg S, Dallongeville J. Fruit and vegetable consumption and risk of coronary heart disease: a meta-analysis of cohort studies. J Nutr. 2006;136(10):2588-93.

53. Siess W, Roth P, Scherer B, Kurzmann I, Bohlig B, Weber PC. Plateletmembrane fatty acids, platelet aggregation, and thromboxane formation during a mackerel diet. Lancet. 1980;1(8166):441-4.

54. Del Turco S, Basta G, Lazzerini G, Evangelista M, Rainaldi G, Tanganelli P, et al. Effect of the administration of n-3 polyunsaturated fatty acids on circulating levels of microparticles in patients with a previous myocardial infarction. Haematologica. 2008;93(6):892-9.

55. Krekels JPM, Verhezen PWM, Henskens YMC. Platelet aggregation in healthy participants is not affected by smoking, drinking coffee, consuming a highfat meal, or performing physical exercise. Clin Appl Thromb Hemost. 2019; 25:1076029618782445

56. Rusca A, Di Stefano AF, Doig MV, Scarsi C, Perucca E. Relative bioavailability and pharmacokinetics of two oral formulations of docosahexaenoic acid/ eicosapentaenoic acid after multiple-dose administration in healthy volunteers. Eur J Clin Pharmacol. 2009;65(5):503-10.

57. Madsen T, Christensen JH, Toft E, Aardestrup I, Lundbye-Christensen S, Schmidt EB. Effect of intravenous omega-3 fatty acid infusion and hemodialysis on fatty acid composition of free fatty acids and phospholipids in patients with end-stage renal disease. JPEN J Parenter Enteral Nutr. 2011;35(1):97-106.

58. Srivastava KC. Docosahexaenoic (C22:6 omega 3) and linoleic acid are antiaggregatory and alter arachidonic acid metabolism in human platelets. Prostaglandins Leukotrienes Med. 1985;17:319-27.

59. Cao J, Schwichtenberg KA, Hanson NQ, Tsai MY. Incorporation and clearance of omega-3 fatty acids in erythrocyte membranes and plasma phospholipids. Clin Chem. 2006;52(12):2265-72.

60. von Schacky C. Prophylaxis of atherosclerosis with marine omega-3 fatty acids. A comprehensive strategy. Ann Intern Med. 1987;107(6):890-9.

61. Mason RP, Libby P, Bhatt DL. Emerging mechanisms of cardiovascular protection for the Omega-3 fatty acid Eicosapentaenoic acid. Arterioscler Thromb Vasc Biol. 2020;40(5):1135-47.

62. McEwen BJ, Morel-Kopp MC, Chen W, Tofler GH, Ward CM. Effects of omega-3 polyunsaturated fatty acids on platelet function in healthy subjects and subjects with cardiovascular disease. Semin Thromb Hemost. 2013;39(1):25-32.

\section{Publisher's Note}

Springer Nature remains neutral with regard to jurisdictional claims in published maps and institutional affiliations.

\section{Ready to submit your research? Choose BMC and benefit from:}

- fast, convenient online submission

- thorough peer review by experienced researchers in your field

- rapid publication on acceptance

- support for research data, including large and complex data types

- gold Open Access which fosters wider collaboration and increased citations

- maximum visibility for your research: over $100 \mathrm{M}$ website views per year

At BMC, research is always in progress.

Learn more biomedcentral.com/submissions 